\title{
A CONSTRUCTION OF NONSTANDARD UNISERIAL MODULES OVER VALUATION DOMAINS
}

\author{
BARBARA L. OSOFSKY
}

A uniserial module over a ring (with 1) is a module whose submodules are linearly ordered by inclusion. A valuation ring is a commutative ring which is uniserial as a module over itself, and a valuation domain is a valuation ring without (nonzero) zerodivisors. If $D$ is a valuation domain with quotient field $Q(D)$, a uniserial $D$-module $U$ is called standard if $U$ embeds in a quotient $Q(D) / J$ where $J$ is a $D$-submodule of $Q(D)$. If there is no such embedding, $U$ is called nonstandard.

Kaplansky conjectured that every valuation ring is a quotient of a valuation domain. Nonstandard uniserials were initially studied to get a counter-example to this conjecture. Given a valuation domain $D$ and a divisible uniserial $D$-module $U$, the ring $A$ with additive group $D \oplus U$ and $(d, u)\left(d^{\prime}, u^{\prime}\right)=\left(d d^{\prime}, d u^{\prime}+u d^{\prime}\right)$ is a valuation ring. Fuchs and Salce [F-Sa] show that $A$ is not a quotient of a valuation domain if (and only if) $U$ is nonstandard.

Proof of the existence of a nonstandard uniserial module $U$ was the most difficult part in obtaining the counterexample to Kaplansky's conjecture. Cyclic and countably generated uniserial modules are always standard, and an almost maximal valuation domain (proper quotients of the domain are topologically complete in the ideal topology) has no nonstandard uniserials. Moreover, every proper submodule of a uniserial module must be standard, so getting nonstandard uniserials requires being able to get embeddings on 'arbitrarily large' submodules of a uniserial $U$, but none of those embeddings can extend to all of $U$.

Previous proofs that nonstandard uniserial modules exist have used extra set theoretic axioms and/or model theoretic techniques. Shelah [S] was the first to show that nonstandard uniserials exist.

\footnotetext{
Received by the editors October 31, 1990 and, in revised form, December 26, 1990.

1980 Mathematics Subject Classification (1985 Revision). Primary 13A18, 13 L05.

Key words and phrases. Valuation domains, uniserial modules, nonstandard uniserials.
} 
He used forcing and absoluteness in model theory. Fuchs and Salce [F-Sa] use the axiom $\diamond_{\omega_{1}}$ and Franzen and Göbel [F-G] the axiom $2^{\aleph_{1}}>2^{\aleph_{0}}$ to get a construction of nonstandard uniserials. Fuchs and Shelah [F-Sh] use a model theoretic argument and the Fuchs-Salce result to conclude that the existence of divisible nonstandard uniserial modules is actually a consequence of the usual axioms of set theory, ZFC. Their methods give no idea of what the valuation domains are nor how to actually get the nonstandard uniserial modules. Bazzoni and Salce in [B-S1] and [B-S2] study quotients of nonstandard uniserials, first looking at quotients of a given nonstandard uniserial and then at which uniserials can arise as quotients of other uniserials. Their construction, based on the construction in [F-Sa], uses $\diamond_{\omega_{1}}$ to give examples of a variety of different kinds of nonstandard uniserials. Eklof [E] extends the Fuchs-Shelah arguments to show that $\diamond_{\omega_{1}}$ is not necessary to prove existence of the kinds of nonstandard uniserials constructed in [B-S2].

This paper constructs any possible kind of nonstandard uniserial modules directly, without any need for extra axioms independent of the usual set theory axioms ZFC, and without the use of model theory. The set theory used is basic material in the study of axiomatic set theory, having as upper bound on the level of unfamiliarity an easy to prove Pressing-Down Lemma on stationary sets which we state below.

Since even stating the main theorem in all its generality involves some messy notation, in this announcement I will give the construction only in a special case. The valuation domain is the same one used in [F-Sa] to get their counterexample to Kaplansky's conjecture, but the nonstandard uniserial does not require $\diamond_{\omega_{1}}$ for its definition. I will then state the complete theorem and try to give some idea how its proof differs from the special case.

THE CONSTRUCTION IN A SPECIAL CASE

Let $\Gamma$ be the free abelian group on the set $\omega_{1}$,

$$
\Gamma=\sum_{\alpha<\omega_{1}} \alpha \mathbf{Z}
$$

Order $\Gamma$ antilexicographically, that is, if $\gamma=\sum_{i=1}^{m} \alpha_{i} z_{\alpha_{i}}$ and $\delta=$ $\sum_{j=1}^{n} \alpha_{j} u_{\alpha_{j}}$ are in $\Gamma$, then $\gamma<\delta$ if and only if $z_{\alpha}<u_{\alpha}$ for $\alpha$ the largest element of $\omega_{1}$ where $\gamma$ and $\delta$ have different projections. 
For $\alpha \in \omega_{1}$, let $\alpha^{\prime}$ denote the element of $\Gamma$ which has projection 0 on each term of the direct sum except for the $\alpha$ th where it has projection 1 . Let $K$ be the field of real numbers or any other uncountable field.

There is a well known construction of a maximally complete valuation domain (every quotient is topologically complete in its ideal topology) with value group $\Gamma$ and residue field $K$. See for example [Kr] where this is referenced to a 1907 paper of $\mathrm{H}$. Hahn. Take 'long formal power series'

$$
\widehat{R}=\left\{\sum_{\delta \in \Delta} k_{\delta} X^{\delta} \mid\left\{k_{\delta}\right\} \subseteq K, \Delta \text { a well ordered subset of } \Gamma^{+} \cup\{0\}\right\}
$$

where $\Gamma^{+}$is the positive cone of $\Gamma$. The operations of addition, multiplication, and taking inverses of these long series are essentially the same as for ordinary power series. As in the case of ordinary power series, any family $\left\{x_{\beta} \equiv x\left(\bmod I_{\beta}\right) \mid\left\{I_{\beta}\right\}\right.$ ideals of $\left.\widehat{R}\right\}$ of finitely satisfiable congruences modulo ideals of $\widehat{R}$ has a solution in $\widehat{R}$.

The support of an element $r=\sum_{\delta \in \Delta} k_{\delta} X^{\delta} \in \widehat{R}$, denoted supp $(r)$, is the set of all $\gamma \in \Gamma^{+} \cup\{0\}$ for which $k_{\gamma} \neq 0$. In addition, in our special case we have a projection support, denoted p-supp, defined by

$$
\begin{aligned}
\operatorname{p}-\operatorname{supp}(r)=\{ & \alpha<\omega_{1} \mid(\exists \gamma \in \operatorname{supp}(r)) \\
& \left.\left(\gamma \text { has nonzero projection on } \alpha \mathbf{Z} \subset \oplus_{\beta<\omega_{1}} \beta \mathbf{Z}\right)\right\} .
\end{aligned}
$$

The valuation domain $R$ we will work with is the smallest valuation subring of $\widehat{R}$ containing all polynomials (elements of finite support) in $\widehat{R}$. This is the valuation domain which is used in [FSa] together with a construction of a nonstandard uniserial over it using $\diamond_{\omega_{1}}$ to get a counterexample to Kaplansky's conjecture.

Let $\widehat{E}$ denote the $R$-module

$$
\widehat{E}=Q(\widehat{R}) / \widehat{R} .
$$

For $M \subseteq \widehat{E}$ and $r=\sum_{\gamma} k_{\gamma} X^{\gamma} \in \widehat{R}$, let $\operatorname{trunc}_{M}(r)$ denote the truncation

$$
\operatorname{trunc}_{M}(r)=\sum_{M X^{\gamma} \neq 0} k_{\gamma} X^{\gamma}
$$

where we simply ignore the terms in the series for $r$ which annihilate $M$. We observe that trunc ${ }_{M}(r)$ is a 'canonical' representation 
of $r$ modulo the annihilator $(0: M)$ of $M$, and $\operatorname{trunc}_{M}(r s)=$ $\operatorname{trunc}_{M}\left(\operatorname{trunc}_{M}(r) \operatorname{trunc}_{M}(s)\right)$.

Observations. The following observations will be used in the sequel, often without specific reference.

(1) For any $r \in R, \mathrm{p}$-supp $(r)$ is finite. If $\varepsilon \in \widehat{R}$ is a unit, then $\mathrm{p}-\operatorname{supp}(\varepsilon)=\mathrm{p}$-supp $\left(\varepsilon^{-1}\right)$.

(2) $\widehat{R}$ and $R$ have the same value group and residue field, so any element of $\widehat{R}$ is of the form $r \varepsilon$ where $r \in R$ and $\varepsilon$ is a unit (constant term $\neq 0$ ) in $\widehat{R}$.

(3) Every element $x$ in the $R$-module $\widehat{E}$ has annihilator of the form $r R$ for some $r \in R$. If $x, y \in \widehat{E}$ have the same annihilator, then there is a unit $\varepsilon \in \widehat{R}$ with $x=y \varepsilon$.

(4) $Q(R) / R$ embeds in $\widehat{E}$, and we identify it with its image in $\widehat{E}$.

(5) Let $x \in \widehat{E}$ and $\varepsilon$ a unit in $\widehat{R}$. Then $x \varepsilon \in x R$ if and only if $\operatorname{trunc}_{x R}(\varepsilon) \in R$.

Forcing truncations of units in $\widehat{R}$ to be in $R$ as in (5) is a crucial ingredient in both our definition of a nonstandard uniserial $V$ and the proof that it is nonstandard.

Construction. For $\alpha<\omega_{1}$, let $U_{\alpha}$ denote the $R$-module

$$
U_{\alpha}=X^{-\alpha^{\prime}} R+R \subset \widehat{E} \text {. }
$$

Note that each $U_{\alpha}$ is cyclic, so by (5), if $\beta<\alpha$ and $\varepsilon_{\alpha}, \varepsilon_{\beta}$ are units of $\widehat{R}$, then $U_{\beta} \varepsilon_{\beta} \subseteq U_{\alpha} \varepsilon_{\alpha}$ if and only if $\operatorname{trunc}_{U_{\beta}}\left(\varepsilon_{\beta} \varepsilon_{\alpha}{ }^{-1}\right) \epsilon$ $R$.

Let $\alpha<\omega_{1}$ and assume we have a family of uniserials $V_{\beta} \subset \widehat{E}$ and units $\varepsilon_{\beta} \in \widehat{R}$ for all $\beta<\alpha$ such that

(a) $\beta>\beta^{\prime} \Longrightarrow V_{\beta} \supset V_{\beta^{\prime}}$,

(b) $\varepsilon_{\beta} \in \widehat{R}$ has p-support $\subseteq\left\{\beta^{\prime} \mid \beta^{\prime}<\beta\right\}$.

(c) $V_{\beta}=U_{\beta} \varepsilon_{\beta}$.

(d) $\varepsilon_{\beta} \notin R$ if $\beta$ is a limit ordinal.

If $\alpha=0$ set $\varepsilon_{\alpha}=1$, and if $\alpha=\beta+1$, set $\varepsilon_{\alpha}=\varepsilon_{\beta}$. Now assume that $\alpha$ is a limit ordinal. We proceed to define $\varepsilon_{\alpha}$ retaining these properties.

Let $\{\mu(\alpha, n) \mid n \in \omega\}$ be an increasing sequence cofinal in $\alpha$. We obtain a unit $\eta_{\alpha} \in \widehat{R}$ with $\left(\bigcup_{\beta<\alpha} U_{\beta}\right) \eta_{\alpha}=\bigcup_{\beta<\alpha} V_{\beta}$ by setting

$$
\eta_{0}=\varepsilon_{\mu(\alpha, 0)} \text {, }
$$


and

$$
\eta_{n+1}=\varepsilon_{\mu(\alpha, n+1)} \operatorname{trunc}_{U_{\mu(\alpha, n)}}\left(\eta_{n} \varepsilon_{\mu(\alpha, n+1)^{-1}}\right) .
$$

This forces $\eta_{n+1}$ and $\eta_{n}$ to have the same initial series outside the annihilator of $U_{\mu(\alpha, n)}$, and $U_{\mu(\alpha, n+1)} \eta_{n+1}=V_{\mu(\alpha, n+1)}$. These initial segments define a unique power series with exponents outside the annihilator of the union of the $U_{\mu(\alpha, n)}$, and $\eta_{\alpha}$ is defined to be that power series. Then $U_{\alpha} \eta_{\alpha} \supset V_{\beta}$ for all $\beta<\alpha$.

Now look at all the coefficients that appear in any $\varepsilon_{\beta}$ for $\beta<\alpha$. There are at most a countable number of them. Hence the subfield $L_{\alpha}$ of $K$ which they generate is countable. Let $\left\{t_{\alpha, n} \mid n \in \omega\right\}$ be any countable subset of $K$ such that

$$
t_{\alpha, n} \notin L_{\alpha}\left(\left\{t_{\alpha, m} \mid m<n\right\}\right) \text {. }
$$

Set

$$
\varepsilon_{\alpha}=\eta_{\alpha} \cdot\left(1+\sum_{n=0}^{\infty} t_{\alpha, n} X^{\mu(\alpha, n)^{\prime}}\right)
$$

We observe using (5) that $U_{\alpha} \varepsilon_{\alpha} \supset U_{\beta} \varepsilon_{\beta}$ for all $\beta<\alpha$, preserving (a). Properties (b) and (d) are clear. Thus transfinite induction gives us a $V_{\alpha}$ for all $\alpha<\omega_{1}$. Set

$$
V=\bigcup_{\alpha \in \omega_{1}} V_{\alpha} \text {. }
$$

Lemma. Let $U_{R} \subseteq \widehat{E}$, and let $\phi: U \longrightarrow \widehat{E}$ be a monomorphism. Then there exists a unit $\varepsilon$ of $\widehat{R}$ such that $\phi(u)=u \varepsilon$ for all $u \in U$. Proof. Let $U=\bigcup_{\beta} u_{\beta} R$ with $\left(0: u_{\beta}\right)=r_{\beta} R$. For any $r \in R$, we observe that

$$
\{u \in \widehat{E} \mid(0: u)=r R\}=\left\{r^{-1} \varepsilon+\widehat{R} \mid \varepsilon \text { a unit of } \widehat{R}\right\} .
$$

Then for all $\beta, \phi\left(u_{\beta}\right)=u_{\beta} \varepsilon_{\beta}$ for some unit $\varepsilon_{\beta} \in \widehat{R}$. If $u_{\gamma}=$ $u_{\beta} r_{\beta, \gamma}$ for some $r_{\beta, \gamma} \in R$, then

$$
u_{\gamma} \varepsilon_{\gamma}=\phi\left(u_{\gamma}\right)=\phi\left(u_{\beta} r_{\beta, \gamma}\right)=u_{\beta} \varepsilon_{\beta} r_{\beta, \gamma}=u_{\gamma} \varepsilon_{\beta},
$$

so $\varepsilon_{\beta} \equiv \varepsilon_{\gamma}\left(\bmod \left(0: u_{\gamma}\right)\right)$ and this system of congruences, being finitely satisfiable, has a solution $\varepsilon \in \widehat{R}$.

Definitions. Let $\mu$ be a cardinal, that is, an initial ordinal. A cub (for closed, unbounded subset) $\mathscr{C}$ in $\mu$ is a subset with the property that $\mathscr{C}$ is not bounded and if $\mathscr{X} \subseteq \mathscr{C}$ and $\sup (\mathscr{X}) \neq \mu$, 
then $\sup (\mathscr{Z}) \in \mathscr{C}$. A subset of $\mu$ is called stationary if it has nonempty intersection with every cub.

The Pressing-Down Lemma. Let $\mu$ be a regular cardinal, and $S \subseteq$ $\mu$ a stationary subset of $\mu$. Let $f: S \longrightarrow \mu$ be a function such that $f(\alpha)<\alpha$ for all $\alpha \in \mu$. Then there exists a $\beta \in S$ such that $f^{-1}\{\beta\}$ is stationary.

See for example, [K, page $80,6.15]$.

Theorem. $V=\bigcup_{\gamma \in \omega_{1}} V_{\gamma}$ is a nonstandard uniserial $R$-module.

Proof. Assume $V$ is standard. By the lemma, there exists a unit $\varepsilon \in \widehat{R}$ such that $V=\left(\bigcup_{\alpha \in \omega_{1}} U_{\alpha}\right) \varepsilon$. By $(5), r_{\alpha}=\operatorname{trunc}_{U_{\alpha}}\left(\varepsilon_{\alpha} \varepsilon^{-1}\right) \epsilon$ $R$ and so has finite p-support. Let

$$
\sigma_{\alpha}=\max \left(\mathrm{p}-\operatorname{supp}\left(r_{\alpha}\right)\right), \quad \sigma: S \longrightarrow \omega_{1}, \quad \alpha \mapsto \sigma_{\alpha}
$$

where $S$ is the stationary set of limit ordinals of $\omega_{1}$. Then $\sigma_{\alpha}<\alpha$ for all $\alpha \in S$, so by the Pressing-Down Lemma, there is a $\beta \in \omega_{1}$ such that

$$
I_{\beta}=\left\{\alpha<\omega_{1} \mid \sigma_{\alpha}=\beta\right\}
$$

is stationary in $\omega_{1}$.

For this $\beta$, we can find an increasing sequence $\left\{\gamma_{n} \mid n<\omega\right\} \subseteq$ $I_{\beta}$ with $\beta<\gamma_{0}$. Denote its supremum in $\omega_{1}$ by $\alpha$. As in the construction, set $\eta_{0}^{\prime}=\varepsilon_{\gamma_{0}}$ and $\eta_{n+1}^{\prime}=\varepsilon_{\gamma_{n+1}} \operatorname{trunc}_{v_{\gamma_{n}}}\left(\eta_{n}^{\prime} \varepsilon_{\gamma_{n+1}}{ }^{-1}\right)$. Then $\eta^{\prime}=\lim _{n \rightarrow \infty}\left(\eta_{n}^{\prime}\right)$ is a unit in $\widehat{R}$ with $\left(\bigcup_{n<\omega} U_{\gamma_{n}}\right) \eta^{\prime}=$ $\left(\bigcup_{n<\omega} U_{\gamma_{n}}\right) \eta_{\alpha}$, and every coefficient of the product $\left(\eta^{\prime}\right)^{-1} \eta_{\alpha}$ is in the field generated by the coefficients of $\left\{\varepsilon_{\gamma} \mid \gamma<\alpha\right\}$. Also,

$$
\begin{aligned}
\eta_{n+1}^{\prime} \varepsilon^{-1} & =\left(\varepsilon_{\gamma_{n+1}} \varepsilon^{-1}\right) \operatorname{trunc}_{v_{\gamma_{n}}}\left(\eta_{n}^{\prime} \varepsilon_{\gamma_{n+1}}^{-1}\right) \\
& =\varepsilon_{\gamma_{n+1}} \varepsilon^{-1} \operatorname{trunc}_{v_{\gamma_{n}}}\left(\left(\eta_{n}^{\prime} \varepsilon^{-1}\right)\left(\varepsilon_{\gamma_{n+1}} \varepsilon^{-1}\right)^{-1}\right)
\end{aligned}
$$

so by finite induction p-supp $\left(\operatorname{trunc}_{v_{y_{n}}}\left(\eta_{n}^{\prime} \varepsilon^{-1}\right)\right)$ is bounded above by $\beta$ for all $n<\omega$, so p-supp $\left(\operatorname{trunc}_{\left(\cup_{n<\omega} v_{\gamma_{n}}\right)}\left(\eta^{\prime} \varepsilon^{-1}\right)\right)$ is bounded above by $\beta$. 
We now have

$$
\begin{aligned}
r_{\alpha} & =\operatorname{trunc}_{U_{\alpha}}\left(\varepsilon_{\alpha} \varepsilon^{-1}\right)=\operatorname{trunc} \\
& =\left(\operatorname{trunc}_{\left(\bigcup_{\gamma<\alpha} U_{\gamma}\right)}\left(\varepsilon_{\alpha} \varepsilon^{-1}\right)\right. \\
& \left.\left(\eta^{\prime} \varepsilon^{-1}\right)\right)\left(\eta_{\alpha}\left(\eta^{\prime}\right)^{-1}\right)\left(1+\sum_{n<\omega} t_{\alpha, n} X^{\mu(\alpha, n)^{\prime}}\right) .
\end{aligned}
$$

The coefficient of $X^{\mu(\alpha, n)^{\prime}}$ in the product of the last two factors is the necessarily nonzero sum of $t_{\alpha, n}$ plus an element in the field generated by the coefficients of the $\varepsilon_{\gamma}$ with $\gamma<\alpha$ and the previous $t_{\alpha, m}$. Hence the p-support of the product of the last two factors contains $\{\mu(\alpha, n) \mid n<\omega\}$. Since $\beta<\alpha=\sup (\{\mu(\alpha, n)\})$, there is an $n<\omega$ with $\beta<\mu(\alpha, n)$. Since the p-support of the truncation is bounded by $\beta$, the p-support of the entire product must also contain $\{\mu(\alpha, k) \mid k>n\}$. But this says that $\mathrm{p}$-supp $\left(r_{\alpha}\right)$ is infinite, so $r_{\alpha}$ cannot belong to $R$, a contradiction.

\section{NOTATION AND STATEMENT OF THE MAIN THEOREM}

The collection of all cyclic submodules of the quotient field $Q(D)$ of a valuation domain $D$ forms a totally ordered group $\Gamma$ called the value group of $D$. For $q \in Q(D)$, the value of $q$, denoted $v(q)$, is the cyclic submodule $q D \in \Gamma . D$ is precisely the set of elements in $Q(D)$ with nonnegative value. Any ideal $I$ of $D$ corresponds to a filter on the nonnegative elements $\Gamma^{+} \cup\{0\}$ of $\Gamma$. Specifically, $I=\left\{d \in D \mid v(d) \in I^{\prime}\right\}$ where $I^{\prime} \subseteq \Gamma^{+}$ satisfies $\alpha \in I^{\prime}, \gamma \in \Gamma^{+}$, and $\gamma>\alpha$ imply $\gamma \in I^{\prime}$. Any uniserial $D$-module $U$ has annihilators of elements of the form $q I$ for some ideal $I \subseteq D$ and $q \in Q(D)$. $U$ is generated by some set $\left\{u_{\alpha} \mid \alpha \in \Delta\right\}$ where $\Delta$ is a well ordered increasing subset of $\Gamma^{+}$and the annihilator of $u_{\alpha}$ is of the form $d_{\alpha} I$ with $v\left(d_{\alpha}\right)=\alpha$. The module $J=\sum_{\alpha \in \Delta} d_{\alpha}{ }^{-1} D \subseteq Q(D)$ and the ideal $I$ are basic data for $U$, and we say that $U$ is of type $[J / I]$.

Let $S^{\prime}=\left\{\gamma \in \Gamma \mid \gamma+I^{\prime}=I^{\prime}\right\}$ and set $\bar{\Gamma}=\Gamma / S^{\prime} . S^{\prime}$ is a convex subgroup of $\Gamma$, and the quotient $\bar{\Gamma}$ is a totally ordered abelian group. Let $\bar{\Delta}$ be the image of $\Delta$ in $\bar{\Gamma} . S=\left\{d \in D \mid v(d) \in S^{\prime}\right\}$ is a multiplicatively closed subset of $D$, and $U$ is a nonstandard $D$-module of type $[J / I]$ if and only if $J=J D_{\underline{S}}$ and $U$ is also a nonstandard $D_{S}$-module of type $[J / I]$. If $\frac{S}{\Delta}$ has countable cofinality, then $U$ of type $[J / I]$ must be standard. That is about all you can say in general. 
Theorem. Let $\Gamma$ be a totally ordered abelian group, $\Delta$ a wellordered increasing subset of $\Gamma^{+}, I^{\prime}$ a filter in $\Gamma^{+} \cup\{0\}$. Set $S^{\prime}=\left\{\gamma \in \Gamma \mid \gamma+I^{\prime}=I^{\prime}\right\}, S=\left\{d \in D \mid v(d) \in S^{\prime}\right\}, \bar{\Gamma}=\Gamma / S^{\prime}$, and $\bar{\Delta}$ the image of $\Delta$ in $\bar{\Gamma}$. Assume $\bar{\Delta}$ has cofinality the successor $\aleph^{+}$of a regular cardinal $\aleph$. Then there exists a valuation domain $D$ with value group $\Gamma$ such that $D$ has a nonstandard uniserial of type $[J / I]$, where $J=\sum_{v(d) \in \Delta} d^{-1} D_{S}$ and $I=\left\{d \in D \mid v(d) \in I^{\prime}\right\}$.

The differences between the proof in our special case and the proof of the general theorem are more technical than conceptual. The change from $\omega_{1}$ to the successor of an arbitrary regular cardinal is minor. It involves replacing 'finite' by 'less than the regular cardinal' in the definition of $R$, and acknowledging limit ordinals smaller than that cardinal in the construction and proof of the theorem. If $\Gamma$ is an arbitrary totally ordered abelian group there is no concept of p-support, so we have some messy technical details that are simply observations in the special case I look at here. Arbitrary $I$ also introduces a smaller quantity of technical details. In addition, to work with an arbitrary $\Delta \subseteq \Gamma$, I must consider two cases which require slightly different proofs at one point. These cases correspond to whether or not a nonstandard uniserial of type $[J / I]$ has any standard quotients.

The minimal number of generators of any uniserial module must be a regular cardinal. The problem with uncountable regular cardinals $\aleph$ that are not successors of regular cardinals is finding some way to insure that the uniserials looked at generated by fewer than $\aleph$ elements are standard without simultaneously forcing the module $V$ being constructed to be standard.

\section{REFERENCES}

[B-S1] S. Bazzoni and L. Salce, On non-standard uniserial modules over valuation domains and their quotients, J. Alg. 128 (1990), 292-305.

[B-S2] S. Bazzoni and L. Salce, Elongations of uniserial modules over valuation domains, J. Alg. (to appear).

[E] P. Eklof, A transfer theorem for non-standard uniserials, Proc. Amer. Math. Soc. (to appear).

[F-G] B. Franzen and R. Göbel, Nonstandard uniserial modules over valuation domains, Results in Math. 12 (1987), 86-94.

[F-Sa] L. Fuchs and L. Salce, Modules over valuation domains, Lecture Notes in Pure and Applied Math., vol. 97, Marcel Dekker, New York, 1985.

[F-Sh] L. Fuchs and S. Shelah, Kaplansky's problem on valuation rings, Proc. Amer. Math. Soc. 105 (1989), 25-30. 
[K] K. Kunen, Set theory: an introduction to independence proofs, North Holland, New York, 1980.

[Kr] W. Krull, Allgemeine Bewertungstheorie, J. Reine Angew. Math. 167 (1932), 160-196.

[S] S. Shelah, Nonstandard uniserial module over a uniserial domain exists, Around Classification theory of Models, Lecture Notes in Math, vol. 1182, Springer-Verlag, Berlin, Heidelberg, New York, 1986, pp. 135-150.

[Sc] O. F. G. Schilling, The theory of valuations, Math surveys, vol. 4, Amer. Math. Soc., Providence, RI, 1950.

Department of Mathematics, Rutgers University, New Brunswick, New JERSEY 08903

E-mail address: OSOFSKY@MATH.RUTGERS.EDU 
